\title{
Componentes pedagógico- didácticos en el diseño y funcionamiento de un centro de escritura digital en educación media
}

Volumen 6 N. ${ }^{\circ} 47$ julio - diciembre de 2019 ISSN: 0122-4328 ISSN-E: 2619-6069 pp. 79-96

\author{
Pedagogical-didactic \\ Components in \\ the Design and \\ Operation of a \\ Digital Writing \\ Center in Secondary \\ Education
}

\author{
Componentes \\ pedagógico- \\ didáticos na \\ concepção e \\ operação de um \\ centro de escrita \\ digital no ensino \\ médio
}

Gerzon Yair Calle Álvarez

Fecha de recepción: 02-02-2019

Fecha de aprobación: 31-05-2019

PARA CITAR ESTE ARTÍCULO

Calle Álvarez, G. Y. (2019). Componentes pedagógico-didácticos en el diseño y funcionamiento de un centro de escritura digital en educación media. Nodos y Nudos, 6(47). https://doi.org/10.17227/nyn.vol6.num47-9583

Licenciado en Español y Literatura, Magíster en Educación, Doctor en Educación de la Universidad de Antioquia. Profesor de cátedra e integrante del grupo de investigación: Didáctica y Nuevas Tecnologías de la misma universidad. gerzon.calle@udea.edu.co http://orcid.org/0000-0002-4083-6051 


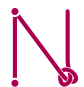

Volumen 6 N. ${ }^{\circ} 47$ julio - diciembre de 2019 ISSN: 0122-4328 ISSN-E: 2619-6069 pp. 79-96

\section{RESUMEN}

Este artículo es el resultado de una investigación sobre los componentes pedagógico-didácticos para el diseño y funcionamiento de un centro de escritura digital (CED) en la educación media. El método utilizado fue el estudio de casos. Participaron dos instituciones educativas -una pública y otra privada-, que ofrecian educación media, en Medellín, Colombia. Se plantearon seis componentes generales, pero en su desarrollo se logró identificar las relaciones entre los referentes pedagógicos y didácticos, sustentados en la práctica e investigaciones previas. Se aplicó el análisis de contenido, apoyado en la herramienta Atlas.Ti. En conclusión, la alfabetización digital se convierte en un eje integrador de los componentes pedagógico-didácticos del CED en el proceso de fortalecimiento de la escritura académica, en los estudiantes de educación media.

Palabras clave: centro de escritura; didáctica; educación media; pedagogía

\section{ABSTRACT}

This article is the result of an investigation on the pedagogical-didactic components for the design and operation of a digital writing center (CED, in Spanish) in secondary education. The method used was the case study. Two educational institutions - one public and one private - that offered secondary education participated in Medellin, Colombia. Six general components were proposed, but in its development, it was possible to identify the relationships between pedagogical and didactic references, based on previous research and practice. Content analysis was applied, supported by the Atlas.Ti tool. In conclusion, digital literacy becomes an integrating axis of the pedagogical-didactic components of CED in the process of strengthening academic writing in middle school students.

Key words: writing center; didactics; secondary education; pedagogy

\section{RESUMO}

Este artigo é o resultado de uma investigação sobre os componentes pedagógico-didáticos para o design e operação de um centro de escrita digital (CED) no ensino médio. 0 método utilizado foi o estudo de caso. Duas instituições de ensino - uma pública e uma privada que ofereciam ensino médio em Medellin, Colômbia participaram. Seis componentes gerais foram propostos, mas em seu desenvolvimento foi possivel identificar as relações entre os referenciais pedagógicos e didáticos, com base na prática e em pesquisas anteriores. A análise de conteúdo foi aplicada, suportada pela ferramenta Atlas.Ti. Concluindo, a alfabetização digital se torna um eixo integrador dos componentes pedagógico-didáticos do CED no processo de fortalecimento da escrita acadêmica em alunos do ensino médio.

Palavras-chave: centro de escrita; didática; ensino médio; pedagogia 


\section{Introducción}

Un centro de escritura (CE) es una estrategia pedagógica que aporta a configurar una actitud epistémica de la escritura en estudiantes y profesores a través del currículo escolar. Palacio (2010) y Stueart (2012) afirman que, en Estados Unidos, desde la década del ochenta, numerosas escuelas de secundaria y media han implementado $C_{\text {, }}$ buscando responder a los retos de escritura propios de estos niveles educativos. Vale anotar que loS CE nacieron en el contexto de la educación superior.

Levin (1989) plantea que los objetivos y filosofía de un CE en educación media incluyen: a) promover el trabajo entre pares; b) atención individual a los estudiantes en los procesos de escritura, c) aportar a las metas y horizonte institucional; d) atraer a los estudiantes que presentan desempeño regular en la escritura para que asistan a los servicios del centro; e) apoyar la institución en el mejoramiento de pruebas de medición institucional. Estos propósitos responden a retos que siguen siendo vigentes en el contexto colombiano, además, están orientados a lograr que el CE permanezca en el tiempo dentro de la institución y que las inversiones económicas que se brinden desde los administrativos se vean reflejados en la calidad de los aprendizajes de los estudiantes, la cual, a su vez, repercutirá en la proyección institucional.

Palacio (2010) realizó una investigación que tenía el propósito de identificar cómo un ce afectaba los niveles de confianza de los estudiantes e influir en sus actitudes hacia la escritura. Este estudio, llevado a cabo en Pace High School, Estados Unidos, partió de una revisión bibliográfica al respecto y se basó en entrevistas a tres estudiantes beneficiarios del $C_{E}$, antes y después de la tutoría académica, y a dos tutores del CE. Las conclusiones señalan que cuando los estudiantes programan su tiempo para trabajar con los tutores, sin presiones externas, se observa un cambio mayor en los grados de confianza sobre la propia escritura; además, cuando esto se adquiere, se está en la capacidad de orientar los procesos de producción de otros estudiantes.

Un CE aporta a las perspectivas de enseñanza de la escritura como proceso, y de esta a través del currículo. Cuando un estudiante participa en alguno de los servicios de un $\mathrm{CE}_{1}$ debe comprender que no asiste a un espacio de corrección textual; por el contrario, encuentra que el texto es una excusa para mejorar su proceso de producción y que estas acciones lo incorporan en los discursos que se abordan dentro de las áreas. Igualmente, un CE va más allá de identificar errores ortográficos y gramaticales, está enfocado en aportar a la formación de mejores escritores.

Dean (2010) documentó los desafíos de los tutores pares del CE de Lakeside High School, Estados Unidos. Estos hacian referencia a cómo el estudiante tutor asumía su rol frente a los compañeros de la institución. Se aplicó un estudio de casos, con diseño etnográfico. Para la recolección de información se implementaron entrevistas individuales, entrevistas de grupos focales, análisis de documentos y observaciones a catorce tutores pares del CE. El autor concluye que los estudiantes evitaban que fueran llamados "profesores", debido a que este calificativo está asociado en la institución a la instrucción directiva, distinta a la posición colaborativa que se buscaba establecer durante las tutorias. Los resultados proporcionaron evidencia de que los tutores pares perciben el curso de preparación como formativo para el acompañamiento de la escritura y favorece la identidad del tutor.

Brinkley (1987) argumenta que las instituciones de educación secundaria y media necesitan más lOS CE que las universidades, debido a que los profesores de estos niveles tienen menor tiempo de acompañar a los estudiantes en el proceso de escritura. Al instaurarse un CE en la educación básica o media se pretende que los estudiantes fortalezcan sus prácticas de lectura y escritura de los discursos académicos y sociales, lo que se verá reflejado en sus aprendizajes a lo largo de la vida. Si un estudiante ingresa a la educación superior con sus procesos de producción e interpretación acordes a ese nivel escolar, tendrá mayores probabilidades de ser exitoso en su vida profesional, social y laboral.

Los propósitos de implementar un CE en la educación media están enfocados en promover las prácticas de escritura académica de las áreas entre los estudiantes y profesores, creando estrategias de ejecución. Sin embargo, ofrecer tutoría académica a los estudiantes es un valor distintivo de estos espacios. Por eso, en este artículo se da a conocer el estudio 
realizado sobre los componentes pedagógico-didácticos involucrados en el diseño e implementación de un centro de escritura digital (CED) para el fortalecimiento de las prácticas de escritura académicas en educación media. Para ello, a continuación, cada sección corresponde a los componentes pedagógico-didácticos, integrando lo teórico, identificado en la revisión de la literatura, y lo práctico, desde la experiencia en la investigación.

\section{Referentes metodológicos}

Para responder a la pregunta de investigación "¿Cuáles son los aportes de dos experiencias de implementación de un centro de escritura digital en los componentes pedagógico-didácticos, orientados al fortalecimiento de la escritura académica en la educación media?", se planteó un estudio de casos para el que se seleccionaron dos instituciones educativas de la ciudad de Medellín, Colombia. El caso 1 corresponde a una institución educativa pública que ofrece educación media técnica y tiene en su política institucional la incorporación de las tecnologías de la información y la comunicación (Tic) en los procesos de enseñanza y aprendizaje, y el mejoramiento de las competencias comunicativas en sus estudiantes. Por otra parte -el caso $2-$, se trata de una institución educativa privada que brinda educación media con énfasis en pedagogía, proyecta en sus estudiantes una formación humanista y entre sus programas de trabajo cuenta con una línea asociada al lenguaje.

Con el objetivo de determinar y hacer seguimiento a los componentes pedagógico-didácticos de lOS CED, para este estudio de casos se plantearon tres fases:

a. Preactiva. Se realizó una exploración de CED en el contexto nacional e internacional, y un reconocimiento de la infraestructura tecnológica de las instituciones educativas. Para la recolección de la información en esta fase, se recurrió a fichas bibliográficas sobre investigaciones previas y fichas de práctica, que contenían una descripción de las características de los CED identificados.

b. Interactiva. Se conformó un equipo colaborador para el montaje y acompañamiento técnico del CED, compuesto por una doctora en Educación, un ingeniero de sistemas y el investigador. Vale anotar que se contó con el apoyo del Programa de Integración de las Tecnologías a la Educación, de la Universidad de Antioquia (Colombia). Además, se desarrolló el pilotaje del CED en ambas instituciones educativas. Para el registro del proceso, el investigador hizo apuntes a modo de bitácora, la cual contempló aspectos pedagógicos, didácticos, técnicos e institucionales.

c. Posactiva. Corresponde a los resultados. Para ello, se analizó cómo operaron los componentes pedagógico-didácticos del CED en ambas instituciones, a partir de la información obtenida durante todo el seguimiento. Para el procesamiento de la información se recurrió a la herramienta Atlas.Ti. Se partió de la información recolectada en cada una de las fases, con sus respectivos instrumentos, teniendo en cuenta que la información obtenida con cada instrumento contribuiría a la comprensión de los componentes pedagógico-didácticos de los CED.

Para la presentación de los resultados se consideró el diseño del CED como propuesta pedagógica para la promoción y fortalecimiento de la escritura académica, la participación de los servicios de cada caso y la descripción teórica y práctica de los componente pedagógico-didácticos que soportaron la propuesta:

\section{Presentación del centro de escritura digital y participación}

El CED buscó responder a los propósitos de lOS CE, tal como fueron planteados inicialmente por North (1984) quien afirma que estos últimos deben aportar a formar mejores escritores, no mejores escritos. El CED tuvo como eje los estudiantes, por ello, en las fases de diseño y funcionamiento se reconoció la diversidad social, lingüística y cognitiva de los usuarios. No habia un prototipo de usuario definido previamente, el estudiante que por iniciativa o remisión de un profesor asistió a los servicios del CED era orientado en su proceso de escritura. Desde estas disposiciones, los profesores/tutores generaron las condiciones didácticas, disciplinares y sociales necesarias para acercar al estudiante a sus procesos cognitivos y metacognitivos para el aprendizaje de la escritura académica. 
En ambos casos se diseñaron e implementaron una serie de servicios para la promoción de la escritura en los estudiantes, pero cada institución educativa determinó la manera de utilizar el recurso. En la tabla 1, se presentan los servicios y el número de estudiantes que lo usaron, y en la tabla 2, está la información de los profesores.

Inicialmente se diseñaron los servicios del CED para ser ofertados virtualmente; sin embargo, debido a las dinámicas administrativas de las instituciones educativas, las dificultades de acceso a internet de los estudiantes desde sus hogares y los retos en la formación de la educación virtual, fue necesario ampliar las posibilidades para la atención presencial. Esta situación se detalla en las tablas 1 y 2. Lo anterior, implicó dimensionar cada componente pedagógico-didáctico, desde su accionar en la presencialidad y la virtualidad, manteniendo sus aportes al fortalecimiento de la escritura académica.

Tabla 1. Servicios y participación estudiantes CED

\begin{tabular}{|c|c|c|c|c|c|c|}
\hline \multirow{2}{*}{ Servicio } & \multicolumn{2}{|c|}{ Total } & \multicolumn{2}{|c|}{$\begin{array}{l}\text { Caso } 1: \text { Institución } \\
\text { educativa pública }\end{array}$} & \multicolumn{2}{|c|}{$\begin{array}{l}\text { Caso 2: Institución } \\
\text { educativa privada }\end{array}$} \\
\hline & $n$ & $\%$ & $n$ & $\%$ & $n$ & $\%$ \\
\hline $\begin{array}{l}\text { Tutoría con tutor virtual en la } \\
\text { plataforma Moodle }\end{array}$ & 13 & 3,8 & 5 & 2,2 & 8 & 7,2 \\
\hline Tutoría con tutor presencial & 48 & 14,1 & 39 & 17,0 & 9 & 8,1 \\
\hline $\begin{array}{l}\text { Taller de escritura en grupo en la } \\
\text { plataforma Moodle (virtual) }\end{array}$ & 64 & 18,8 & 33 & 14,4 & 31 & 27,9 \\
\hline Taller de escritura en grupo presencial & 91 & 26,8 & 66 & 28,8 & 25 & 22,5 \\
\hline $\begin{array}{l}\text { Biblioteca de recursos digitales para } \\
\text { escribir mejor }\end{array}$ & 124 & 36,5 & 86 & 37,6 & 38 & 34,2 \\
\hline Total & 340 & 100,0 & 229 & 100,0 & 111 & 100,0 \\
\hline
\end{tabular}

Fuente: elaboración propia.

Tabla 2. Servicios y participación profesores CED

\begin{tabular}{|c|c|c|c|c|c|c|}
\hline \multirow{2}{*}{ Servicio } & \multicolumn{2}{|c|}{ Total } & \multicolumn{2}{|c|}{$\begin{array}{l}\text { Caso } 1 \text { : Institución } \\
\text { educativa pública }\end{array}$} & \multicolumn{2}{|c|}{$\begin{array}{l}\text { Caso 2: Institución } \\
\text { educativa privada }\end{array}$} \\
\hline & $n$ & $\%$ & $n$ & $\%$ & $n$ & $\%$ \\
\hline Asesoria virtual & 1 & 4,0 & 0 & 0,0 & 1 & 5,3 \\
\hline Asesoria presencial & 24 & 96,0 & 6 & 100,0 & 18 & 94,7 \\
\hline Total & 25 & 100,0 & 6 & 100,0 & 19 & 100,0 \\
\hline
\end{tabular}

Fuente: elaboración propia. 


\section{Componente 1: entorno virtual de aprendizaje para el soporte del centro de escritura digital}

Un entorno virtual de aprendizaje facilita, primero, la conectividad para el intercambio de contenidos entre sujetos que se encuentran distantes (Farías y Montoya, 2009) y, segundo, las actividades de aprendizaje a través de internet. Además, reproduce o complementa las condiciones de una clase presencial, a la vez que proporciona a los profesores y estudiantes mecanismos de comunicación e interacción, aunque suprime las condiciones espaciales y temporales (Cabrera, 2014; Silva y Romero, 2014). Sin embargo, el entorno virtual de aprendizaje se diferencia de una página web por su enfoque formativo y porque su diseño responde a las investigaciones sobre la representación y uso de la información para el aprendizaje (Silva y Romero, 2014).

En el caso específico del CED, como entorno virtual de aprendizaje, se identificaron los siguientes propósitos formativos: promover la escritura académica a través de las áreas del currículo escolar, reconocer que la escritura es un proceso, apoyar la formación de mejores escritores, aportar a las metas institucionales de calidad educativa y al aprendizaje de la escritura académica de las instituciones. En ese sntido, se diseñaron y operaron los servicios del CED en las dos instituciones contempladas en este estudio. Por ello, la estructura y rutas de navegación se consolidaron a partir de cómo los estudiantes aprendian, representaban la información que se les brindaba y construían nuevas ideas.

Por otro lado, Edel, Garcia y Tiburcio (2008), y Farías y Montoya (2009) afirman que las TIC, según sus caracteristicas, facilitan los procesos pedagógicos, debido a las alternativas de formación individualizada, planificación del aprendizaje, estructura abierta y modular, comodidad e interactividad. Sin embargo, el entorno virtual de aprendizaje por sí solo no genera los procesos del pensamiento y lenguaje necesarios en la producción textual. Diseñar y operar un CED desde la plataforma Moodle, debía considerar esas caracteristicas para la gestión pedagógica y didáctica en la orientación de las prácticas de escritura académica.

A partir de estos planteamientos, se determinó crear el entorno virtual de aprendizaje bajo la plataforma Moodle (figura 1). El diseño del CED desde Moodle estaba orientado a facilitar la conectividad, es decir, que los estudiantes, profesores y tutores hicieran uso de los servicios, sin necesidad de compartir un mismo espacio físico a la vez. Para ello, los participantes del CED tenían un usuario y contraseña, y por medio de la página web de las instituciones educativas, lograban conectarse, siempre y cuando tuvieran un dispositivo tecnológico con acceso a internet. Esta característica de Moodle se reflejó en el CED en las dos instituciones, al otorgarles a los usuarios acceso y herramientas, según sus necesidades y ritmos de aprendizaje.

En ambos casos, el cED ofreció una serie de servicios virtuales para el acompañamiento y fortalecimiento de la escritura académica: a) talleres, tutorias, y guías de autoaprendizaje para estudiantes; b) asesorias, talleres, foros de discusión, recursos para la enseñanza de la escritura desde las áreas, para profesores; c) recursos para el acompañamiento de la escritura desde las áreas y foros de discusión, para profesores y tutores. Los servicios se diseñaron y operaron pensando en las oportunidades de estudiantes o profesores para trabajar de manera autónoma o con el acompañamiento de uno de los tutores del CED.

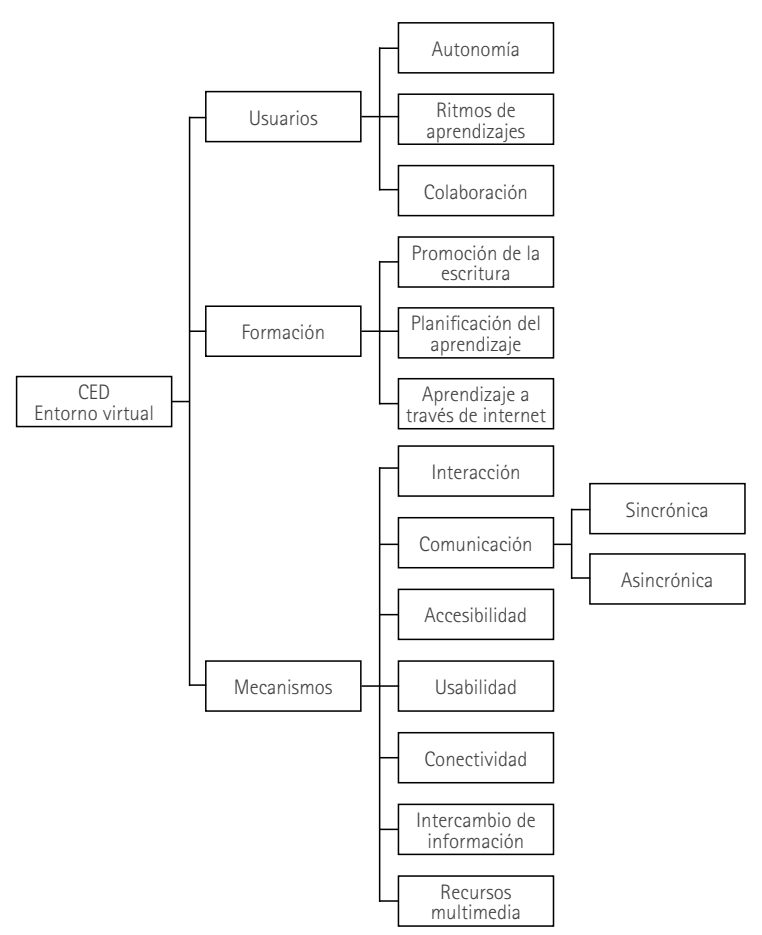

Figura 1. Componente entorno virtual de aprendizaje gestor del ced

Fuente: elaboración propia. 
Se encontró que, desde el CED, los estudiantes solicitaron la tutoría académica para apoyar la resolución de tareas de escritura asignadas en las áreas del currículo escolar o se apoyaron de alguna de las guías de escritura alojadas en la biblioteca de recursos. Esa posibilidad de conectividad permitió que los estudiantes y profesores accedieran a recursos didácticos de manera autónoma o dirigida. Por ejemplo, un estudiante del caso 1, que requería hacer un comentario en un blog de la clase de filosofía, accedió a la biblioteca de recursos del CED y descargó una guía que le ofrecía, de manera didáctica, las orientaciones para la planeación, producción, revisión y publicación del comentario.

Las interacciones generaron acciones sociales alrededor de la promoción de la escritura académica en las instituciones. En los talleres virtuales, que se ofrecieron en los dos casos, había foros de discusiones sobre los problemas de escritura de algún texto académico, y contaron con la participación de estudiantes, profesores y tutores. En sus discursos es posible reconocer las relaciones que establecen y las estructuras del conocimiento de los participantes. Como lo plantean Ávila y González-Álvarez (2016), las tIc pueden asociarse a las literacidades, por tanto, un entorno virtual para la escritura académica puede aprovechar esa relación.

El diseño del CED posibilitó la comunicación sincrónica y asincrónica de los participantes. Los estudiantes tenían recursos para el trabajo autónomo y social, lo que exigía que se establecieran entre ellos mecanismos de comunicación. En el caso 1, el servicio de tutoría virtual se implantaba por medio del chat de Moodle, lo que proporcionaba una comunicación sincrónica. Igualmente, en el caso 2, los talleres virtuales estaban diseñados para que las estrategias de acompañamiento y devolución de la tarea de escritura se diera de manera asincrónica, por medio de foros y respuesta al ejercicio que los estudiantes subian a la plataforma. Esto adquiere connotaciones importantes, como las señaladas por Farías y Montoya (2009), sobre el entorno virtual de aprendizaje como un espacio social, y las de Gros y Contreras (2006), para quienes, en la sociedad del conocimiento, la comunicación a través de medios electrónicos es importante para la formación de las competencias ciudadanas.
Por ello, aunque en el CED existían servicios orientados a atender los estudiantes de manera individual, se buscó que se reconociera que los diálogos e intercambios de información alrededor del texto del estudiante eran un proceso social orientado al aprendizaje. Igualmente, se plantearon estrategias de aprendizaje con un enfoque social, con el propósito de generar cercanía entre los participantes y reconocer las potencialidades de los otros que les aportan en la construcción de sus ideas. En los dos casos, hubo estudiantes en los talleres virtuales que, antes de subir la tarea de escritura, la ponian a consideración de los compañeros del taller, para que estos realizaran comentarios sobre aspectos positivos o por mejorar del escrito; con esta información el estudiante aplicó las acciones respectivas para mejorar su producción y reconocía sus aspectos positivos para replicarlos en futuros escritos académicos.

Mayer (2005) y Rudolph (2017) plantean tres principios de aprendizaje multimedia: a) el sistema de procesamiento de la información de los seres humanos incluye los canales visuales y auditivo; b) cada canal tiene una capacidad limitada, y c) el procesamiento activo involucra aspectos cognitivos coordinados que se dan durante el aprendizaje. Estos principios implicaron en el CED el uso de recursos multimedia para la orientación, acompañamiento y aprendizaje de la escritura académica, respondiendo a criterios de congruencia entre la imagen, los audios, el texto, no abusando de los recursos visuales y diseñando rutas para el acceso y manejo de la información. El uso de la multimedia sirvió como complemento para el desarrollo de estrategias de exploración, planeación, producción, revisión y divulgación de las prácticas de escritura académica de los estudiantes y profesores; esto generó oportunidades para la reflexión sobre la tarea de escritura.

\section{Componente 2: escritura académica en una cultura digital}

Actualmente los estudiantes se encuentran inmersos en una cultura de lo digital, pero ello no necesariamente implica que estén alfabetizados digitalmente (Gutiérrez y Tyner, 2012; Valverde y Caro, 2015). Si bien participan en redes sociales, intercambian mensajes por dispositivos móviles, se les dificulta trasladar 
sus conocimientos informales sobre uso de las TIC a espacios de escritura académica, a pesar de las destrezas que adquieren para su uso con fines lúdicos, se evidencian dificultades para su aplicación en estrategias como elaborar esquemas de sus propias ideas, elaborar un resumen, leer críticamente (Carlino, 2013). En cambio, cuando un estudiante se apoya en las Tic para su producción textual, estas se convierten en soportes del trabajo académico. La propuesta de CED planteó que los servicios y recursos que se encontraran en el entorno virtual fortalecerian prácticas de escritura académica en los estudiantes.

En particular, se observan dificultades para el uso de los recursos digitales para apoyar o fortalecer la escritura en estudiantes de educación media. En esta dirección, la propuesta de CED contempló estrategias para articular procesos de alfabetización digital y alfabetización académica, como ocurre con la exploración de recursos virtuales durante la tutoría académica para reconocer aciertos y desaciertos en los textos. De este modo, se buscaba promover las prácticas de escritura académica apoyadas por TIC, como una actividad situada en un contexto social, específicamente en los discursos de las áreas del currículo escolar. Misas (2017) afirma que los problemas de lectura y escritura de los estudiantes que ingresan a la educación superior, generan deserción y frustración en la universidad.

Los enfoques metodológicos para orientar la escritura académica fueron: la escritura como proceso y la escritura a través del currículo. Esto implicaba comprender que la producción de textos académicos respondía a formas de construcción de conocimientos propios de las áreas. Por ello, la orientación y acompañamiento de la escritura en el CED no se concebian como asuntos remediales, sino principalmente epistémicos.

De acuerdo con Tchudi y Yates (1983) y con el trabajo de Bazerman et al. (2016), el movimiento de escribir a través del currículo en la educación básica y media se caracteriza porque las áreas de estudio se encuentran menos vinculadas a las disciplinas que en la universidad, el ámbito de actividad está definido por los espacios sociales de las áreas y, además, las prácticas lectoras están orientadas a las necesidades en las aulas de la escuela primaria y secundaria; es decir, se enfatiza en habilidades como la extracción de información, la identificación de la idea principal y el uso del razonamiento inferencial. El CED se pensó para aportar a la lectura y escritura de las diversas áreas del currículo escolar, reconociendo que los discursos que se tejen dentro de ellas tienen referentes semejantes como el desarrollo del aprendizaje. Los recursos y servicios ofrecidos por el CED consideraron la escritura como proceso, para lo cual se incorporaba el desarrollo de habilidades cognitivas y metacognitivas para la construcción planificada y consciente de la tarea de escritura.

Teniendo en cuenta los planteamientos del grupo Didactext (2003) sobre la importancia de tener en cuenta las características procesuales, controlables, educables y flexibles de las estrategias cognitivas y metacognitivas, en el acompañamiento de producción textual del CED se reconocieron las fases de la escritura y posibles estrategias para aplicar en cada una de ellas: a) en la planificación, para la elaboración de esquemas mentales y la ordenación de las acciones a desarrollar; b) durante la producción, para el trabajo en género discursivo, textualización y recursos multimodales; c) en la revisión, mediante la lectura consciente y la corrección de la producción.

En el diseño y funcionamiento del CED se consideraron, también, las posibilidades de las tIC como herramientas para pensar y actuar solos y con otros, en correspondencia con los postulados de Coll, Mauri y Onrubia (2008), para quienes las tic son herramientas psicológicas mediadoras en la enseñanza y aprendizaje, entre las relaciones de los participantes, los contenidos de aprendizajes y la comunicación. El CED, como mediador en el acompañamiento de las prácticas de escritura académica de los estudiantes, dependía de los usos que le daban los tutores y los estudiantes a los recursos y servicios que alli se ofrecian, desde las posibilidades del trabajo autónomo y las interacciones entre los participantes, para la planificación, producción y revisión de las tareas. Esto se evidenció en los talleres virtuales donde los alumnos utilizaban la plataforma del CED para planear, crear y revisar un texto, pero, en todo el proceso podía apoyarse en el tutor o intercambiar ideas con sus pares. 
El CED proponía y orientó actividades de escritura académica auténtica; situación que permitió en el estudiante el desarrollo de procesos de pensamiento que conectaran las actividades de escritura con situaciones significativas del mundo académico y social. Las temáticas planteadas en los talleres y biblioteca de recursos eran relevantes para los estudiantes y profesores, lo que repercutía en la motivación para el acceso a los recursos y servicios que se encontraron en el CED. En las instrucciones para la participación de las actividades de escritura o tutorías, se sugirió al estudiante que tuviera algún tipo de conexión con las actividades de las áreas, concursos de escritura, publicaciones o eventos de la ciudad donde ellos tuvieran intenciones de participar.

Rangel (2011) afirma que saber leer y escribir no es suficiente para que las personas se desempeñen en la sociedad actual, es necesario el desarrollo de habilidades para participar en la cultura digital. Asi mismo, Cassany (2000) plantea que si se quiere que la didáctica de la escritura siga respondiendo a los usos sociales y que el estudiante aprenda, se deben reconocer los cambios en la enseñanza de la escritura en un entorno digital. Por ello, en el CED la alfabetización académica y digital se complementaron; es decir, no fue suficiente acompañar las prácticas de escritura académica, también fue necesario orientar al estudiante en la búsqueda, selección, uso y valoración de la información que se encontraba en los soportes digitales. Los estudiantes debian asumir una actitud crítica, reflexiva y activa en la interacción con la información y soportes digitales, que le aportaron a la producción textual.

A partir de lo anterior, en el CED se abordaron las escrituras analógica y digital, pues se consideró que ambos tipos de escritura conviven actualmente en la sociedad y en la escuela, y que ninguna es más importante que la otra, cada una requiere procesos cognitivos y metacognitivos específicos. Por ejemplo, dentro de la biblioteca de recursos digitales los estudiantes podían encontrar una guía para responder exámenes escritos y otra para escribir colaborativamente una wiki. La figura 2 presenta las relaciones del componente de las prácticas de escritura académica en una cultura digital.

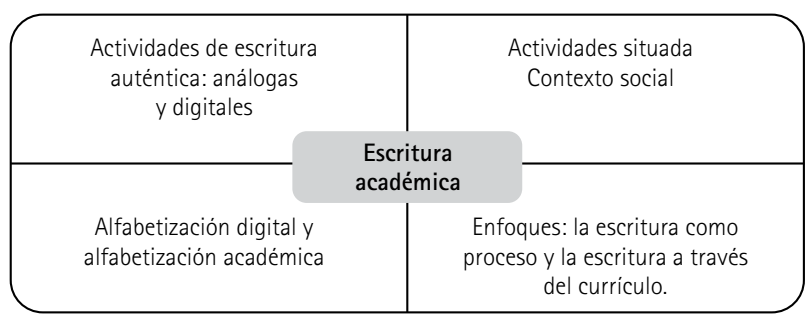

Figura 2. Componente escritura académica y TIC Fuente: elaboración propia.

\section{Componente 3: formación de mejores escritores}

La figura 3 presenta las características del componente de formación de mejores escritores.

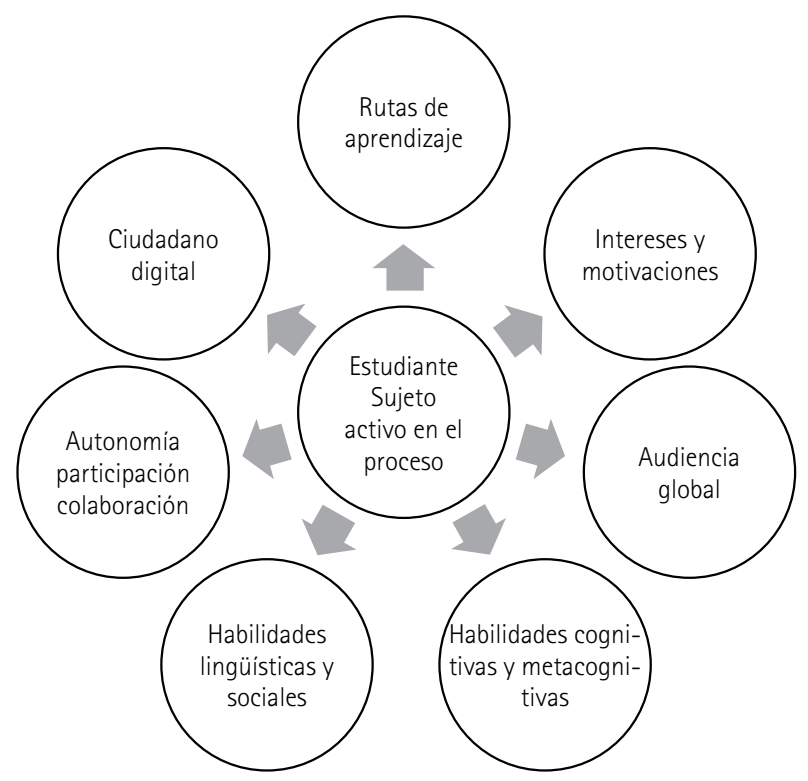

Figura 3. Componente formación de mejores escritores

Fuente: elaboración propia.

De acuerdo con Bazerman et al. (2016), la escritura y el pensamiento de los estudiantes avanzan mejor cuando desarrollan las actividades de escritura desde sus intereses y propios interrogantes, sin temor a ser penalizados. En ese sentido, el CED buscó activar el interés del estudiante por la escritura académica. Para ello, se partió de situaciones de las dinámicas institucionales o situaciones de aula, se sugirió que para participar en los talleres virtuales de escritura 
los estudiantes tuvieran un motivo para escribir, ya fuera una tarea escolar o una situación personal. Activar el interés de los estudiantes fue un reto no solo para el diseño de los recursos y servicios del CED, sino también para que ellos accedieran y los usaran. Al acceder al CED por un interés personal, los alumnos tenían mayores posibilidades de participar activamente durante todo el servicio.

El CED propuso contenidos que les permitieron a los estudiantes explorar, comentar, revisar, construir conceptos y relaciones, desde problemas sociales y académicos pertinentes para el aprendizaje. El estudiante diseñó las rutas de aprendizaje en la producción textual, es decir, desde sus necesidades iniciales, experiencias y saberes previos, determinó cuál servicio o recurso es el que le aportaba en la solución a su problema. Además, cuando el estudiante tomaba la decisión, desde los servicios del CED se orientó para que en la tarea de escritura experimentará y aplicara la información que tenía y la que se le brindó. Un estudiante de once, del caso 1, expresa: "Por medio del CED nos guiamos para hacer mejor nuestro trabajo académico".

Uno de los intereses del CED es que los estudiantes produjeran un texto que fuera compartido con una audiencia global, que no se limitara a conseguir una nota, la lectura del profesor o a la socialización del aula. De acuerdo con Rincón, Sanabria y López (2016), quien escribe debe estar en la capacidad de pensar en escenarios que le aporten al texto. En esta línea, en ambos casos, cuando un texto tenía las condiciones de calidad para ser publicado, los estudiantes mantenían los derechos de producción, sin embargo, se les invitó a que lo compartieran en el espacio de divulgación del CED, las carteleras institucionales, eventos de ciudad, publicaciones regionales, actos cívicos y culturales, espacios virtuales académicos, como redes sociales de divulgación escolar, redes de comunicación escolar. Se buscó que los estudiantes identificarán que sus producciones tenían las condiciones de calidad para ser compartidas y leídas con una audiencia más amplia, además, se les aclaró que cuando un texto se publicaba estaba propenso a la reflexión y crítica de los lectores, y que estas eran un aporte para el propio análisis sobre el proceso de escritura.
Rincón, Sanabria y López (2015) plantean que, en la escritura, la autorregulación se presenta en la autonomía del escritor durante la resolución del problema y la toma de decisiones, para lo cual traza una meta, monitorea y autoevalúa el desarrollo del texto. Los servicios del CED se orientaron a que los estudiantes fueran autónomos durante la escritura, desarrollaran la autorregulación, reconocieran sus formas de aprender e identificaran los errores en su ejercicio. En ambos casos, cuando los estudiantes y profesores asistían a un taller virtual o leian una guía de escritura durante la producción textual, encontraron estrategias que los invitaron a planear la tarea de escritura, revisar el propio texto y autoevaluar su proceso y su producto escritural. Lograr que el estudiante desarrollara estrategias de autorregulación se convirtió en un objetivo, debido a su valor en la activación de habilidades cognitivas y lingüísticas en el aprendizaje de la escritura.

En tanto se considera que el estudiante es un sujeto activo en el aprendizaje de la escritura académica, en el CED no se le escribía o corregía el texto, él debía asumir su compromiso con la escritura, para lo cual podía preguntar al profesor/tutor y generar estrategias de autorrevisión y corrección. Por ello, podía asistir a una tutoría sobre un texto académico y el trabajo desarrollado durante esta podía ser suficiente para comprender la ruta a seguir o para saber cuáles recursos virtuales del CED utilizar para la producción, sin necesidad de regresar a otra tutoría. El estudiante activo aplicó sus saberes previos, se interrogó sobre el texto y el uso de la lengua durante el proceso de construcción del texto. Al respecto, Shobeiri (2016) afirma que las TIC instituyeron nuevas metodologías en la búsqueda de un estudiante activo responsable de su propio aprendizaje.

Coll (2004) plantea que una de las características de los entornos apoyados en tic es la interactividad, la cual favorece la adaptación a distintos ritmos de aprendizajes. En el CED, se partió de las diferencias cognitivas, sociales y biológicas de los estudiantes en el proceso de orientación de la escritura, el reconocer dificultades o potenciales para el aprendizaje, edad, motivos para asistir al CED, todos estos considerados aspectos diferenciadores al momento de desarrollar un servicio, por lo cual algunas acciones requirieron 
tiempos distintos según los estudiantes. Por ejemplo, aun cuando los talleres virtuales estaban pensados para que se resolvieran en tres horas, llegaron a permanecer abiertos hasta tres semanas para que los estudiantes los terminaran. No se buscó cantidad, era más importante la calidad en el acompañamiento y el aprendizaje del propio proceso de escritura.

Cabero y Llorente (2008) sugieren que los sujetos deben saber utilizar las nuevas herramientas de comunicación que se encuentran disponibles en la sociedad del conocimiento, lo que implicará la alfabetización en diferentes códigos, sistemas simbólicos, y formas de interactuar con la información. Por ello, el CED aportó a la consolidación de ciudadanos digitales; los estudiantes se enfrentaron a diversos formatos de textos gráficos, visuales, audiovisuales, hipermediales, hipertextuales, lo que les implicó hacer diferentes tipos de lectura, comprender las estructuras textuales y extraer la información que se requería. Además, durante la escritura de un texto, fue imperativo que buscaran, accedieran, analizaran, evaluaran y produjeran, desde las posibilidades de los recursos digitales que les ofrecía el CED, páginas web o plataformas educativas.

\section{Componente 4: acompañamiento en la producción textual}

Para guiar a los estudiantes en la planeación, producción y revisión de sus propios textos, el profesor/tutor ejemplificó, discutía, comentaba, facilitaba, apoyaba, mostraba rutas de trabajo. En ningún caso actuó como corrector o revisor de estilo. Además, se comprendió que el profesor/tutor no era la única fuente de información, en cambio, este proponía al estudiante rutas y mecanismos para acceder y comprender la información que requería en el proceso de producción del texto, sobre aspectos de composición o recursos de información sobre las áreas.

En la investigación de Werner (2013) se reconocen como positivas para el ejercicio de la tutoría académica las actividades de formación que se brinda a los tutores por parte de los CE. Se tuvo presente que los profesores/tutores no podían quedar solos o aislados, era importante crear condiciones de encuentro para el diálogo, revisión, planeación y acciones de mejora de los servicios y recursos del CED. Además, la tutoría académica no era una actividad en solitario, por el contrario, era una responsabilidad colectiva. Aunque, a los profesores/tutores se les asignaba la prestación de un servicio, durante su ejecución recibían apoyo de otro profesor/tutor, o en las actividades académicas los profesores/tutores ampliaron o profundizaron en la tarea de escritura.

Cuando se ofrecía los servicios a los estudiantes, los profesores/tutores planearon desde los recursos que se disponían en el CED, para ello, se tenían presentes los aprendizajes que los estudiantes desarrollaban a lo largo de la vida: seleccionar los recursos y las estrategias de aprendizajes que fueran detonantes de procesos cognitivos y comunicativos; reconocer los referentes didácticos y disciplinares según los productos textuales esperados; generar estrategias de aprendizajes colaborativos, autónomo o multimedia, según el servicio y la población beneficiada. Sin embargo, los profesores/tutores eran conscientes de que la planificación inicial era una proyección; es decir, durante la prestación del servicio de tutoría las dinámicas que se daban entre los estudiantes, el profesor, el texto y los recursos digitales, determinarian las estrategias didácticas a desarrollar.

Según Seoane y García (2007), para construir un entorno de aprendizaje social, no es suficiente con habilitar un foro, es fundamental que los participantes se encuentren bien liderados. Desde esta posición en el CED, el profesor/tutor tenía la responsabilidad de participar en los procesos de comunicación, plantear pautas de trabajo, fomentar la participación de los estudiantes y orientar la producción textual. Además, se reafirmó la idea que el aprendizaje es una responsabilidad compartida, donde el estudiante, el profesor/tutor y el entorno virtual del CED median en la promoción y mejoramiento de las prácticas de escritura académica del estudiante. Un estudiante de undécimo, del caso 1, expresó que una ventaja del CED fue "el acompañamiento y el desarrollo de diferentes actividades a través de la tecnología".

Los recursos y servicios del CED no se limitaron a la escritura analógica; también se incorporaron actividades de escritura digital, teniendo en cuenta los planteamientos de Sánchez-Rosete (2012), Chaverra (2012) y Calle (2014), quienes expresan que la escritura digital 
tiene una complejidad en la producción distinta al texto análogo. Por ello, los profesores/tutores mantuvieron una posición didáctica y disciplinar para el acompañamiento en la producción de textos digitales. No se trató de abordar dificultades sobre el uso de herramientas, era necesario orientar al estudiante para que comprendiera las dinámicas sociales de los textos digitales y que reconociera los procesos cognitivos que debía desarrollar durante la planeación, producción y revisión de este tipo de textos como, por ejemplo, las relaciones de concordancia entre las imágenes y las gráficas.

Durante el acompañamiento de un estudiante en la producción de un texto, el profesor/tutor aplicaba planes flexibles; las dinámicas que se establecian durante el servicio no eran estándar, variaban de acuerdo con los intereses de los estudiantes. Esto se evidenciaba en las pautas que se brindaron sobre la tarea de escritura, las rutas que se recomendaron a los estudiantes para explorar los recursos del CED y otros externos. Además, se buscó que las interacciones entre los profesores/tutores y el estudiante se caracterizaran por ser dialógicas, horizontales y colaborativas; permitiendo que el estudiante se mantuviera activo durante la tutoría, la comprensión de sus procesos cognitivos en la producción del texto y favoreciera el aprendizaje entre iguales. La figura 4 presenta las características del componente del profesor/tutor.

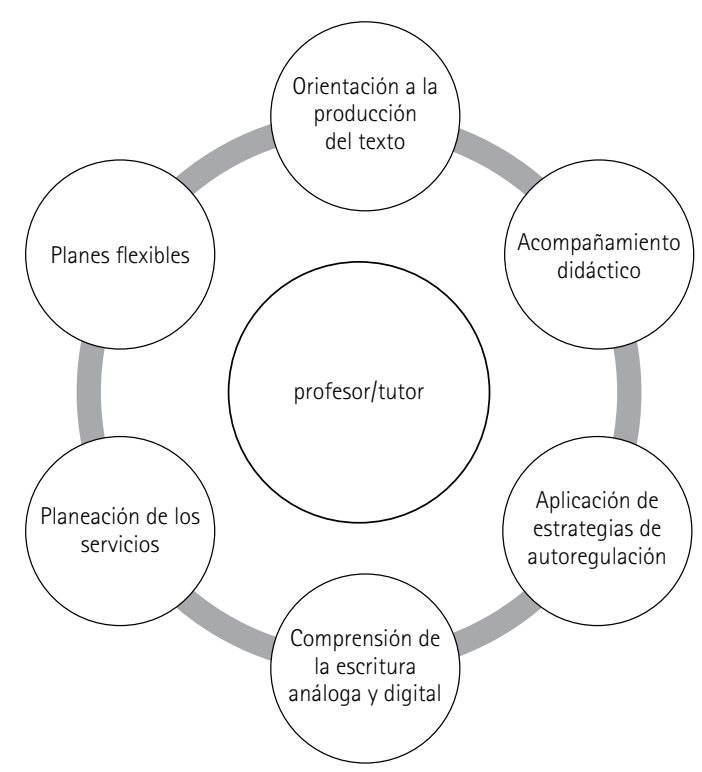

Figura 4. Componente profesor/tutor Fuente: elaboración propia.
Componente 5: recursos digitales para la promoción de la escritura académica

Desde el CED se diseñaron recursos digitales para el aprendizaje de la escritura académica, en concordancia con la investigación de Figueroa, Aillon y Fuentealba (2014), quienes concluyen que el uso de recursos TIC requiere un diseño didáctico que potencie la gestión del conocimiento. Los recursos digitales para la orientación de las prácticas de escritura académica se diseñaron según los criterios vigentes de la didáctica de la escritura, según los propósitos del CED, y desde la exploración de los saberes previos de los estudiantes, que estuvieran asociados a los trabajos académicos que se proponian en las áreas o intereses institucionales. Por ejemplo, en el caso 2, un concurso del cuento, y en el caso 1, los proyectos pedagógicos integradores de la formación técnica.

Cabero y Llorente (2008) consideran que una ventaja de las tic es la cantidad de información a la que tienen acceso los estudiantes, la cual continúa en aumento y mejorando su calidad. El CED, en las dos instituciones, ponía a disposición de los profesores y estudiantes recursos digitales de otros entornos académicos, que fueron revisados y evaluados previamente, desde su pertinencia para el fortalecimiento de la escritura académica en la educación media. Desde la biblioteca de recursos, accedian a páginas web que les presentaban tutoriales de escritura, ejemplos de diferentes tipos de textos.

Cabero y Llorente (2008) afirman que la flexibilidad de las TIC permite a los estudiantes acceder a la información y a la formación en cualquier momento, lugar y ritmo que ellos decidan. También podian descargar los recursos, hacer pausas, ingresar y salir del CED las veces que así lo consideraron. Además, se diseñaron los recursos de manera que fueran fáciles de usar, donde el alumno con unos conocimientos básicos de navegación en plataformas digitales e internet los utilizara. Igualmente, se implementaron íconos estándar de la cultura digital para navegar por el entorno del CED y los recursos, 
por ejemplo, las flechas para indicar direcciones, el subrayado para determinar hipervínculos, un triángulo inclinado a la derecha para representar un texto audiovisual.

Los recursos digitales se diseñaron para operar durante actividades convocadas por el CED, según el recurso; sin embargo, sus usos se ampliaron con la utilización en el aula de clases por parte de los profesores. Esto concuerda con la afirmación de Cacheiro (2011), para quien los recursos TIC son claves para el trabajo centrado en el estudiante y permiten a los profesores que los integren a las prácticas de aula. Los docentes de ambos casos hicieron uso de los recursos del CED para el trabajo de la escritura académica apoyada por tic desde las diferentes áreas o fueron incorporados en proyectos institucionales. Por ejemplo, en el caso 1, se utilizó una de las guías sobre párrafos argumentativos para hacer un taller institucional durante la semana del idioma español; en el caso 2, se incorporaron los talleres virtuales a la cátedra de desarrollo del pensamiento. Lo anterior reflejó que los recursos y servicios del CED fueron adaptados a las necesidades institucionales, donde el CED superó el entorno virtual.

El grupo de investigación Didactext (2003) considera que en el proceso de escritura intervienen la memoria, la motivación-emociones y las estrategias cognitivas y metacognitivas. Estos aspectos se adoptaron en el diseño y selección de los recursos digitales en el CED, para que respondieran a los objetivos de generar motivación e interés de los estudiantes para el desarrollo de la tarea de escritura, proporcionar la información suficiente para que de manera autónoma desarrollaran el texto, guiarlos en su construcción, favorecer procesos cognitivos y metacognitivos. Por ejemplo, en el caso 1, en la guia de escritura de una wiki, los estudiantes identificaban las instrucciones y el paso a paso para participar en la construcción de un recurso wiki; al final de la guía encontraban una serie de preguntas sobre el proceso desarrollado y otros recursos externos para profundizar en la temática. La figura 5 resume las características del componente de los recursos digitales para la promoción de la escritura académica.

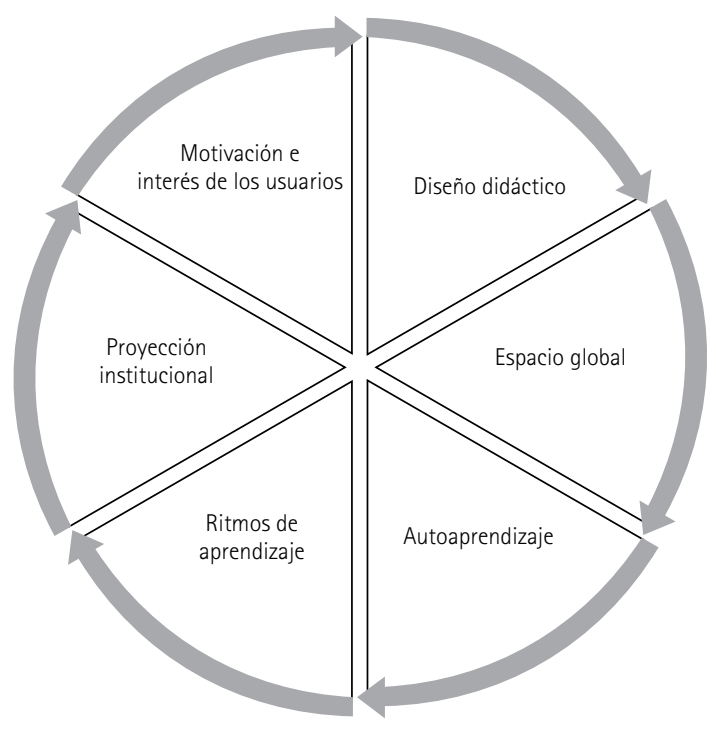

Figura 5. Componente recursos digitales Fuente: elaboración propia.

\section{Componente 6: la evaluación formativa en la tutoría académica}

La evaluación de los procesos de escritura académica en el CED tenía un carácter formativo (figura 6). Para Rosales (2014) en la evaluación formativa durante el proceso de enseñanza se identifican las deficiencias, lo que permite que se tomen decisiones pertinentes y adecuadas para mejorar el aprendizaje. En el CED, los estudiantes y profesores encontraron recursos para el seguimiento, evaluación y autoevaluación de los servicios y la producción de los propios textos académicos. Las estrategias de evaluación formativa que se aplicaron pretendian que el participante concibiera la evaluación como un proceso continuo que le proporcionaba información sobre las estrategias cognitivas y lingüísticas que aplicó durante la producción del texto. Para ello, el profesor/tutor realizó actividades de retroalimentación para el mejoramiento de la tarea de escritura, y lo acompañó en la planeación, producción y revisión del texto.

Al comienzo de cada servicio del CED se aplicó una evaluación de saberes previos, esta ayudó al estudiante a redefinir su conocimiento, construir conceptos y lograr profundidad en los intereses iniciales. Además, lo centró en los objetivos del servicio, contextualizando 
la teoría y la práctica, en la escritura académica. Por otra parte, los profesores/tutores partían de la información para determinar aspectos donde se requería mayor acompañamiento, necesidades de información, redireccionar las estrategias de aprendizaje, ampliar los recursos de aprendizaje. Los saberes previos de los estudiantes eran relevantes para establecer relaciones de interacción entre los conocimientos adquiridos por los estudiantes y los objetivos de aprendizajes propuestos en el CED.

\begin{tabular}{c}
\hline $\begin{array}{c}\text { Evaluación inicial: } \\
\text { saberes previos }\end{array} \quad \begin{array}{c}\text { Evaluación continua: } \\
\text { realimentación }\end{array} \quad \begin{array}{c}\text { Evaluación final: } \\
\text { autoevaluación }\end{array}$ \\
\hline
\end{tabular}

Figura 6. Componente evaluación formativa

Fuente: elaboración propia.

Barberá (2016) afirma que la realimentación es fundamental en la evaluación para el aprendizaje, además, es importante el uso que de ella hagan los estudiantes para avanzar en los conocimientos. En la tutoría académica, la evaluación, aportó a la toma de decisiones del profesor/tutor y del estudiante para orientar y revisar el proceso de escritura. La evaluación se pensó como un asunto coherente entre los propósitos del CED, los intereses del estudiante y el acompañamiento del profesor/tutor, por tanto, se buscó evaluar la producción del texto por parte del estudiante desde la aplicación de habilidades cognitivas, sociales, discursivas. Esto permitía integrar la evaluación y no dejarla de manera aislada o como un asunto que solo se daba al final o sobre el producto; además, permitió que el estudiante hiciera parte de la evaluación.

Igualmente, al final de los servicios del CED, los estudiantes respondían una autoevaluación que tenia como propósito que ellos aplicaran estrategias metacognitivas en la autorrevisión del texto. Las preguntas estuvieron enfocadas en la composición, desde lo lingüístico y textual; buscaban que el estudiante hiciera un ejercicio de verificación y mejoramiento del texto, al tiempo, que le aportara a reconocer aciertos y desaciertos, que le sirvieron para fortalecer su escritura. La autoevaluación estuvo en la dirección del estudiante como sujeto central y activo del aprendizaje de la escritura académica, además, este ejercicio le permitió tener mayor control de la composición y reconocer sus habilidades cognitivas y metacognitivas.

\section{Conclusiones}

El CED, en ambos casos, se presentó como una estrategia pedagógica institucional para promoción de la escritura. Sin embargo, su abordaje didáctico dependió de las decisiones administrativas que se orientaron desde los directivos de la institución y las decisiones de aula por parte de los profesores. Esta situación es comprensible debido a que los CE responden a las filosofías y realidades institucionales.

Un entorno virtual de aprendizaje por sí solo no logrará resolver los problemas de escritura de los estudiantes, sin embargo, si ofrece una serie de posibilidades para el diseño y montaje de un CED, y su incorporación en las instituciones educativas: entornos flexibles de aprendizaje; interacciones entre los estudiantes, profesores, tutores, recursos, texto; recursos interactivos; apoyo a los procesos de evaluación y autoevaluación de los procesos de composición. Diseñar y operar un CED desde Moodle aportó a las dinámicas institucionales y funcionales de los integrantes de la comunidad educativa. Situación que va en correspondencia con la afirmación de Ruíz y Rosales (2017) la importancia de la participación de profesores, alumnos y padres de familia, para la construcción de la escuela.

El uso de la virtualidad para los servicios evidenció que en el caso 1 los estudiantes tomaron la iniciativa y accedieron a la tutoría y los talleres de escritura; sin embargo, los profesores no accedieron a la asesoría virtual. En el caso 2, los profesores accedieron a la asesoría virtual, y los estudiantes hicieron mayor uso de los servicios presenciales. Aunque, estamos en una cultura de lo digital, el uso de los servicios del CED evidenció que en ambos casos hace falta fortalecer la alfabetización digital, en la búsqueda de un mayor nivel de integración de las tecnologías digitales para el aprendizaje y desempeño social.

El diseño del entorno virtual de aprendizaje, en el que se soportó el CED, favoreció el acceso, uso de los recursos digitales de manera autónoma y el trabajo 
colaborativo, por parte de los estudiantes y profesores. Sin embargo, estos beneficios se veían limitados por las dificultades de conexión que tenían los hogares y los retos de formación en la educación virtual, en los estudiantes de la educación media. Por otra parte, las acciones del CED brindaron herramientas académicas y técnicas para la comprensión y producción de textos digitales, así se mantuvo el principio de formar mejores escritores.

Cuando se decide implementar un CE en una institución de educación media, se deberá partir de la realidad institucional, la población beneficiada y la disponibilidad de recursos humanos y económicos; además, hay que proponer una filosofía propia que sea desarrollada, mantenida y reconocida por la comunidad educativa. Lo que concuerda con los postulados de Levin (1989) quien considera que los CE de la escuela media están más enfocados en situaciones prácticas, debido a la poca dotación y financiamiento que se les brinda desde las administraciones de las instituciones, lo que repercute en dificultades para cumplir con los objetivos inicialmente establecidos y su duración en el tiempo.

\section{Referencias}

Ávila, N. y González-Álvarez, P. (2016). Web 2.0 y escritura universitaria: oportunidades para la escritura situada y el aprendizaje colaborativo en un curso online. En D. Riestra, IV Jornadas Internacionales de Investigación y Prácticas en Didáctica de las lenguas y las literaturas (pp. 798-817). Universidad Nacional de Río Negro, Bariloche, Argentina. https://www.researchgate.net/profile/Natalia_Avila_Reyes/publication/305507135_Web_20_y_escritura_universitaria_oportunidades_para_la_escritura_situada_y_ el_aprendizaje_colaborativo_en_un_curso_onlinel links/5792850908aec89db78662ad.pdf

Barberá, E. (2016). Aportaciones de la tecnología a la e-Evaluación. Revista de Educación a Distancia, 50(4), 2-10. http://revistas.um.es/red/article/view/270811

Bazerman, C., Little, J., Bethel, L., Chavkin, T., Fouquette, D. y Garufis, J. (2016). Escribir a través del currículum. Una guía de referencia. Córdoba: Universidad Nacional de Córdoba. https://rdu.unc.edu.ar/bitstream/handle/11086/4030/ Bazerman\%20et\%20al\%20_2016_Escribir\%20a\%20traves\%20de\%20Curriculum.pdf?sequence $=8$ ctisAllowed $=y$

Brinkley, E.H. (1986). Writing centers in secondary schools: An idea beyond the puberty stage. The Writing Lab
Newsletter, 11(4), 5-7. https://wlnjournal.org/archives/ v11/11-4.pdf

Cabero, J.C. y Llorente, M. (2008). La alfabetización digital de los alumnos. Competencias digitales para el siglo xx. Revista Portuguesa de Pedagogía, 42(2), 7-28 http://tecnologiaedu.us.es/cuestionario/bibliovir/jca26.pdf

Cabrera, J.M. (2014) Un objeto virtual de aprendizaje (OVA) para el movimiento armónico simple (MAS) y sus aplicaciones. Entornos, 28, 71-85. https://www.journalusco. edu.co/index.php/entornos/article/view/526/996

Cacheiro, M.L. (2011). Recursos educativos tic de información, colaboración y aprendizaje. Pixel-Bit, Revista de Medios y Educación, (39), 69-81. http://e-spacio.uned.es/fez/ eserv/bibliuned:425-Mlcacheiro-5010/Documento.pdf

Calle, G.Y. (2014). Las habilidades del pensamiento crítico durante la escritura digital en un ambiente de aprendizaje apoyado por herramientas de la web 2.0. Encuentros, 12(1), 27-45. http://repositorio.uac.edu.co/jspui/ bitstream/11619/1442/1/Las\%2Ohabilidades\%20del $\% 20$ pensamiento\%20cr\%C3\%ADtico\%20durante\%20la\%20 escritura.pdf

Carlino, P. (2013). Alfabetización académica diez años después. Revista Mexicana de Investigación Educativa, XvII(57), 355381. http://www.redalyc.org/pdf/140/14025774003.pdf

Cassany, D. (2000). De lo analógico a lo digital. El futuro de la enseñanza de la composición. Lectura y Vida, 21(4), 6-15. http://www.labev.uerj.br/textos/De\%20lo\%20analogic0\%20a\%20lo\%20digital_Daniel\%20Cassany.pdf

Chaverra, D.I. (2012). Las habilidades metacognitivas en la escritura digital. Revista Lasallista de Investigación, 8(2), 104-111. https://www.researchgate.net/profile/ Dora_Chaverra-Fernandez/publication/262457501_ Metacognitive_skills_in_digital_writing/links/555b318b08ae6fd2d829a8b6.pdf

Coll, C. (2004). Psicología de la educación y prácticas educativas mediadas por las tecnologias de la información y la comunicación: una mirada constructivista. Sinéctica, 25, 1-24. http://www.redalyc.org/pdf/998/99815899016.pdf

Coll, C., Mauri, T. y Onrubia, J. (2008). La utilización de las tecnologias en la información y la comunicación en la educación: del diseño tecno-pedagógico a las prácticas de uso. En C. Coll y C. Monereo (coord.), Psicología de la educación virtual (pp. 74-103). Madrid: Morata.

Dean, C. (2010). The ecology of peer tutoring: Perspectives of student staff in one high school writing center. [Tesis de doctorado, Universidad de Maine]. Repositorio institucional UMaine. http://digitalcommons.library.umaine. edu/cgi/viewcontent.cgi?article $=1503$ ctcontext=etd

Didactext (2003). Modelo sociocognitivo, pragmalingüistico y didáctico para la producción de textos escritos. 
Didáctica, Lengua y Literatura, 15, 077-104. http://exordio.qfb.umich.mx/archivos\%20PDF\%20de\%20trabajo\%20UMSNH/Aphilosofia/publicacion.pdf

Edel, R., Garcia, A. y Tiburcio, A. (2008). La modalidad a distancia para la educación tecnológica de posgrado: ¿Es de interés para los egresados? Revista Electrónica lberoamericana sobre Calidad, Eficacia y Cambio en Educación, 6(1), 154-191. http://www.rinace.net/arts/vol6num1/art9.pdf

Farías, G.M. y Montoya, J. (2009). Gestión de un entorno virtual de aprendizaje para el desarrollo de competencias profesionales interculturales: una experiencia de educación superior entre México y España. Apertura, 1(1), 6-19. http://www.udgvirtual.udg.mx/apertura/index. php/apertura/article/view/13/13

Figueroa, B., Aillon, M. y Fuentealba, A. (2014). La escritura académica con soporte de esquemas digitales en la formación docente. Revista de Universidad y Sociedad del Conocimiento (RUSC), 11(1), 18-32. http://dx.doi.org/10.7238/ rusc.v11i1.1665

Gros, B. y Contreras, D. (2006), La alfabetización digital y el desarrollo de competencias ciudadanas, Revista Iberoamericana de Educación, 42, 103-125. http://rieoei.org/rie42a06.htm

Gutiérrez, A. y Tyner, K. (2012). Educación para los medios, alfabetización mediática y competencia digital. Comunicar, 19(38), 31-39. https://www.revistacomunicar.com/ indice-en/articulo.php?numero=38-2012-05

Levin, A.K. (1989). Goals and philosophies of high school writing centers. En P.B. Farrell-Childers (ed.), The high school writing center: Establishing and maintaining one (pp. 2330). Urbana, IL: National Council of Teachers of English. https://wac.colostate.edu/books/hswc/

Mayer, R.E. (2005). Cognitive theory of multimedia learning. En R.E. Mayer (ed.), The Cambridge Handbook of Multimedia Learning (pp. 31-48). Cambridge: Cambridge University Press.

Misas, M.M. (2017). La argumentación como mapa de ruta del proyecto de vida: una experiencia significativa en el aula. Nodos y Nudos, 5(43), 120-132. https://revistas.pedagogica.edu.co/index.php/NYN/article/view/8035/6309

North, S.M. (1984). Revisiting "The Idea of a Writing Center". The Writing Center Journal 15, 17-19.

Palacio, K. (2010) Re-centering Students' Attitudes About Writing: A Qualitative Study of the Effects of a High School Writing Center. [Tesis de maestria, Nova Southeastern University]. Repositorio institucional Nova http:// nsuworks.nova.edu/writing_etd/2

Rangel, A. (2011). Alfabetización digital:el caso de los profesores de la Universidad Autónoma Metropolitana Unidad Cuajimalpa. [Tesis de doctorado, Instituto Politécnico Nacional].
Repositorio institucional upn (en versalitas) http://itzamna.bnct.ipn.mx/bitstream/handle/123456789/11672/alfabetizacion.pdf?sequence $=1$

Rincón, L., Sanabria, L.B. y López, O. (2015). Aproximación a un modelo de autorregulación en escritura académica a partir del análisis de protocolos. Folios, 1(43), 59-76. http://revistas.pedagogica.edu.co/index.php/RF/article/ view/3517

Rosales, M. (2014). Proceso evaluativo: evaluación sumativa, evaluación formativa y Assesment su impacto en la educación actual. En Congreso Iberoamericano de Ciencia, Tecnología, Innovación y Educación. Buenos Aires, Argentina. http://s3.amazonaws.com/academia.edu. documents/45818861/662.pdf?AWSAccessKeyld=AKIAIWOWYYGZ2Y53UL3ACEExpires $=1497226890 \&$ Signature $=91 \mathrm{VgFsNg}$ frkRIOivy0qnYHY\%2B3ig\%3D\&tresponse-content-disposition=inline \%3B\%20filename\%3DEvaluacion_formativa.pdf

Rudolph, M. (2017). Cognitive Theory of Multimedia Learning. Journal of Online Higher Education, 1(2).

Ruiz, M. y Rosales, L. (2017). La Red Lenguaje México. Otra educación es posible. Nodos y Nudos, 5(42), 126-137. https://doi.org/10.17227/nyn.vol5.num42-8035

Sánchez-Rosete, L. (2012). Currículum y escritura digital. En M. Estebanell Minguell, P. Cornellà Canals y D. Codina Regàs (eds.), XX Jornadas universitarias de tecnología educativa (pp. 128-133). Girona, España.

Seoane, A.M. y García, F.J. (2007). Los origenes del tutor: fundamentos filosóficos y epistemológicos de la monitorización para su aplicación a contextos de e-learning. Teoría de la Educación. Educación y Cultura en la Sociedad de la Información, 8(2), 9-30 http://www.redalyc. org/html/2010/201017334002/

Shobeiri, N. (2016). Comunicación y educación: nuevos escenarios en la sociedad del conocimiento. Opción, 32(12), 661-685.

Silva Quiroz, J.E. y Romero, M. (2014). La virtualidad una oportunidad para innovar en educación: un modelo para el diseño de entornos virtuales de aprendizaje. Revista Didasc@lia:Didáctica y Educación, 5(1), 1-22. https://dialnet.unirioja.es/servlet/articulo?codigo $=6584034$

Stueart, K.C. (2012). A Proposal for a Writing Center and a Peer Tutor Training Course at Fayetteville High School. [Tesis de maestría]. Universidad de Arkansas, Estados Unidos. http://scholarworks.uark.edu/cgi/viewcontent. cgi?article $=1284$ ctcontext $=$ etd

Tchudi, S.N. y Yates, J. (1983). Teaching Writing in the Content Areas: Senior High School. Washington: National Education Association Professional Library. p. 80. e http://files. eric.ed.gov/fulltext/ED232212.pdf 
Valverde, M.T. y Caro, M.T. (2015). Desarrollo de la competencia en escritura académica con recursos digitales en el área de comunicación en lengua española. En M.T. Tortosa Ybáñez, J.D. Álvarez Teruel y N. Pellín Buades, XIII Jornadas de Redes de Investigación en Docencia Universitaria: Nuevas estrategias organizativas y metodológicas en la formación universitaria para responder a la necesidad de adaptación y cambio (pp. 1760-1774). Universidad de Alicante, España https:// rua.ua.es/dspace/bitstream/10045/49491/1/XIII_Jornadas_Redes_133.pdf
Werner, C.L. (2013). Constructing Student Learning through Faculty Development: Writing Experts, Writing Centers, and Faculty Resources. CEA Forum, College English Association, 42(2), 79-92. http://files.eric.ed.gov/fulltext/ EJ1018288.pdf 


\section{Diálogo del conocimiento}

Esta nueva lectura del artículo me emocionó más que la primera, seguramente por las situaciones generadas por la crisis sanitaria. En especial, la reflexión frente a las dificultades a las que se han visto enfrentadas las instituciones educativas obligadas por las directrices educativas del gobierno para impartir educación virtual en todos los niveles educativos.

En ese sentido, el texto recobra toda su vigencia en el momento actual. Desafortunadamente, las investigaciones que hacen los maestros, ya sea para obtener su título de pregrado o posgrado, no parecen tener el alcance necesario para cambiar las prácticas pedagógicas. Por esto, es muy importante que los maestros publiquen los trabajos y enriquezcan así el saber pedagógico que ha gestado en las Universidades, los centros de investigación, así como en las prácticas pedagógicas.

En este proceso investigativo, se resalta la rigurosidad teórica y metodológica de la experiencia que la convierte en un aporte pedagógico para afrontar los problemas de escritura en el ámbito escolar porque lo plantean desde la perspectiva de la escritura digital. Por ello, considero que el haber explorado la relación tutor-estudiante les permitió a los maestros crear un ambiente en el que la autonomía y la libertad se convierten en una condición para la producción de texto. De la misma forma, la propuesta de los talleres para entablar una comunicación e interacción tutor-estudiantes, permiten otorgarle a la escritura una perspectiva cultural en tanto hace posible tener en cuenta el contexto y, por ende, los intereses de los estudiantes. Llama la atención la biblioteca de recursos digitales que les ofreció alternativas digitales para que los estudiantes puedan escribir mejor, asi como la promoción la escritura para publicar. Estos mecanismos posibilitan compartir la lectura con otros, asi como aceptar la crítica como aprendizaje para escribir mejor.

El enfoque pedagógico al introducir alternativas de formación individualizada, planificación del aprendizaje con estructura abierta y modular, y también la interactividad, facilitaron a los profesores trabajar desde el interés de los estudiantes, a través de la motivación del trabajo autónomo y social, aspectos que contribuyen a la formación de competencias para ser un ciudadano digital. Finalmente, se resalta la insistencia en plantear la alfabetización digital, teniendo en cuenta que aunque los jóvenes participan en redes sociales intercambiando mensajes por dispositivos móviles, presentan dificultades para trasladar estos usos de la escritura a las formas de escribir exigidas por la escuela, a saber, la escritura académica.

Martha Helena Barreto R. Universidad Distrital 